\title{
TITLE: RISK ASSESSMENT UNDER DEEP UNCERTAINTY: A METHODOLOGICAL COMPARISON
}

Julie Shortridge (corresponding author)

Virginia Tech

Biological Systems Engineering

Blacksburg, Virginia 24061

Email: jshortridge@vt.edu

Telephone: +1-540-231-2797

Terje Aven

University of Stavanger

Centre for Risk Management and Societal Safety

Stavanger, Norway

Email: terje.aven@uis.no

Telephone: +47-5183-2267

Seth Guikema

University of Michigan

Department of Industrial and Operations Engineering

Ann Arbor, Michigan USA

Email: sguikema@umich.edu

Telephone: +1-734-764-6475

Page 1 


\section{ABSTRACT:}

2 Probabilistic Risk Assessment (PRA) has proven to be an invaluable tool for evaluating risks in

3 complex engineered systems. However, there is increasing concern that PRA may not be

4 adequate in situations with little underlying knowledge to support probabilistic representation of

5 uncertainties. As analysts and policy makers turn their attention to deeply uncertain hazards such

6 as climate change, a number of alternatives to traditional PRA have been proposed. This paper

7 systematically compares three diverse approaches for risk analysis under deep uncertainty

8 (qualitative uncertainty factors, probability bounds, and robust decision making) in terms of their

9 representation of uncertain quantities, analytical output, and implications for risk management. A

10 simple example problem is used to highlight differences in the way that each method relates to

11 the traditional risk assessment process and fundamental issues associated with risk assessment

12 and description. We find that the implications for decision making are not necessarily consistent

13 between approaches, and that differences in the representation of uncertain quantities and

14 analytical output suggest contexts in which each method may be most appropriate. Finally, each

15 methodology demonstrates how risk assessment can inform decision making in deeply uncertain

16 contexts, informing more effective responses to risk problems characterized by deep uncertainty.

18 Keywords: Deep uncertainty, probabilities, probability bounds, robust decision making 


\section{INTRODUCTION}

The use of probabilities in describing uncertainty is a foundational pillar of risk analysis. Quantifying the likelihood of undesired consequences in complex systems requires a mechanism for drawing inference and quantifying uncertainty in situations where frequentist data is limited. The usefulness of Bayesian probability in meeting these needs is clear and unparalleled. Nevertheless, it has long been acknowledged that Bayesian probabilities are conditioned on underlying knowledge ${ }^{(1)}$ and that low levels of underlying knowledge can present issues for probabilistic representation of uncertainty. Using a single probability (or probability distribution) to describe uncertainty masks information about what portion of the overall uncertainty is epistemic versus aleatory ${ }^{(2,3)}$ as well as the strength of underlying knowledge supporting that probability ${ }^{(4)}$. Conditions of deep uncertainty, such as situations where probabilities of different outcomes are unknown, previous data is deemed insufficient for estimating future consequences, and experts disagree on the consequences of different policies, present particularly difficult challenges ${ }^{(5)}$. These issues have led to an ongoing, lively discussion regarding the theoretical and practical basis for alternative approaches to uncertainty representation in risk assessment see, for instance, the discussion by Dubois ${ }^{(3)}$ and others in Risk Analysis Vol. 30, No. 3.

Attempts to address high-profile contemporary issues such as climate change have also raised a number of practical issues associated with applying probabilistic risk assessment to these problems. These problems are often characterized by multiple experts, stakeholders, and decision-makers who may all have dramatically different beliefs regarding future uncertain events. A probabilistic analysis may be met with resistance in situations with multiple stakeholders who disagree with the likelihood and consequences assigned by selected experts ${ }^{(6)}$. Some research has found that experts themselves may be hesitant to assign subjective probabilities that may be perceived as unreliable or untrustworthy ${ }^{(7)}$. It has been argued that probabilistic projections of climate change could mislead decision-makers by obscuring the real range of possible futures and implying a greater degree of certainty than actually exists ${ }^{(8)}$, resulting in "disguised subjectivity" (9) when the underlying assumptions and uncertainties are not made clear. This has led to some organizations promoting the use of probabilistic analysis only in very limited cases. For instance, IPCC guidance on reporting climate impacts requires high confidence (based on robust sources of evidence that are in general agreement with each other) for authors to characterize uncertainties probabilistically ${ }^{(10)}$.

Given these concerns, a number of methodologies have been proposed to provide a more comprehensive treatment of non-probabilistic uncertainty, including "frequency of probability" approaches ${ }^{(11)}$; numerical alternatives to probabilities such as imprecise probabilities ${ }^{(12)}$, probability bounds analysis ${ }^{(13)}$, and possibility theory ${ }^{(14)}$; qualitative measures for describing the weight of evidence 
on which probability assessments are based ${ }^{(4)}$; and robustness-based decision support frameworks that do not rely on probabilities such as Robust Decision Making (RDM) ${ }^{(15)}$, Info-Gap Theory ${ }^{(16)}$, and Resilience Analytics ${ }^{(17,18)}$ Existing research on these approaches largely focuses on their development, debate on their practicality and theoretical foundations, and application to specific problems. However, many of these methodologies have been developed in relative isolation from each other, making the advantages, limitations, assumptions and practical implications of each approach relative to others unclear. This limits the degree to which researchers and practitioners can build upon previous research in this field and apply these methods to problems where probabilistic analysis is considered insufficient or inappropriate.

Systematic comparisons between different approaches could serve to address some of these issues. However, relatively few comparisons between different methods exist, and those that do tend to focus on numerical alternatives to probability without considering semi-quantitative and robustness-based approaches. For instance, Dubois and Prade ${ }^{(19)}$ compare Bayesian probabilities, belief functions, and possibility theory in their ability to combine multiple expert opinions, finding that all methods can be subject to numerical instability when faced with strongly conflicting information. Soundappan et al. ${ }^{(20)}$ disagreement and imprecision, comparing their underlying assumptions and treatment of different sources of uncertainty and imprecision. Aven and Zio ${ }^{(6)}$ critically review multiple uncertainty representations as applied to a simplified nuclear reactor failure risk problem and present a broad framework for uncertainty analysis that is compatible with these representations. Similarly, Hall et al.'s ${ }^{(21)}$ comparison of RDM with Info-Gap theory to evaluate greenhouse-gas emission policies focuses on two robustness-based approaches.

The objective of this work is to systematically compare three diverse approaches to risk assessment under deep uncertainty in terms of their representation of uncertain quantities, analytical output, and information provided for risk management and decision making. This work builds on previous comparisons that have generally focused on relatively similar methodologies (for example, alternative uncertainty representations in Aven and $\mathrm{Zio}^{(6)}$ or robustness-based approaches in Hall et al. ${ }^{(21)}$ ) by comparing three methodologies (semi-quantitative uncertainty factors, probability bounds analysis, and Robust Decision Making) that address epistemic uncertainty in very different ways. A comparison of these methodologies also has the added benefit of evaluating two approaches (uncertainty factors and RDM) that are relatively new to the risk and reliability field. The methods selected for this comparison should not be considered a judgement on which methods are most appropriate or promising, and future work comparing additional methodologies would be a valuable extension of this research. 
We use a simple, stylized flood risk example to evaluate the informational requirements, underlying assumptions, and information provided to decision-makers in each case, and also evaluate how each approach relates to fundamental issues associated with risk assessment and description. While the example problem includes a number of simplifications that would make it unsuitable for evaluating flood risk in a real city, the use of a simple problem for methodological comparison does serve a number of purposes that have been highlighted by previous studies employing this approach ${ }^{(6,21-23)}$. One main advantage of using a stylized example problems is to avoid situations where computational challenges overwhelm and obscure fundamental differences between the evaluated methods ${ }^{(23)}$. A simple problem "allows clear focus on the essential comparisons among decision approaches," ${ }^{, 20)}$ rather than the intricacies of the example problem. Similarly, we evaluate each methodology's treatment of one particular form of deep uncertainty (the existence of uncertain model parameters that cannot be described probabilistically), recognizing that other forms may exist (for instance, the presence of disagreement or uncertainty regarding model structure itself). The use of a stylized example problem that focuses on a single form of uncertainty is a logical first step in comparing three very divergent approaches to risk assessment under deep uncertainty. Once the fundamental differences between approaches have been highlighted and clarified, then comparisons using more sophisticated examples and different forms of uncertainty would be a valuable area for additional research. By comparing these methodologies in a clear and comprehensive manner, this paper aims to improve understanding of how the choice of methodology may impact implications for risk management and suggest contexts in which certain approaches may be more suitable than others.

\section{FRAMEWORK FOR COMPARISON}

To provide a clear framework for comparing non-probabilistic and quasi-probabilistic approaches to risk assessment, it is important to be clear about what exactly risk assessment entails. In this analysis, risk assessment refers to an analytical process that aims to identify and describe possible hazards, their causes and consequences, and the uncertainty surrounding their occurrence. In a probabilistic risk assessment, this process includes hazard identification, cause and consequence analysis, and a probabilistic analysis that describes the likelihood of occurrence for different scenarios and their consequences ${ }^{(24)}$. This process ultimately results in a risk description, which can be used alongside managerial review and judgment to inform risk management decisions.

Traditionally, risk has been described using the "triplets" definition of risk introduced by Kaplan and Garrick ${ }^{(11)}$, which included possible scenarios or events $A$, the consequences that result from these events $C$, and the associated probability of an event $P$. Depending on the phenomena of interest, this probability $P$ can represent the frequency of a repeatable event or the Bayesian (subjective) probability of 
1 a unique event ${ }^{(25,26)}$. However, this is not a perfect tool for describing uncertainty, particularly because it 2 provides no information on the background knowledge on which $P$ is based ${ }^{(23)}$. To more explicitly 3 account for this element of uncertainty, we use Aven's ${ }^{(24)}$ more general risk description which includes specific events $A^{\prime}$, a measurement of the quantities of interest $C^{\prime}$ that represent consequences $C$, a measurement $Q$ of the uncertainty in $A^{\prime}$ and $C^{\prime}$, and the knowledge $K$ on which $A^{\prime}, C^{\prime}$, and $Q$ are based. Figure 1 illustrates this conceptualization.

This comparison will assess how each of the three methods relate to the process and resulting risk description described above through the use of a stylized climate change adaptation problem related to flood risks in a riverfront city. The city is considering upgrading its existing floodwall due to concerns that its reliability has decreased with age and that climate change could result in floods occurring more frequently. The outcome quantity of interest is total cost over the next 30 years, which is the sum of damage costs from flooding and construction costs should the floodwall be upgraded. A number of simplifying assumptions are made so that complexities in the example problem do not obscure or confuse the methodological comparison. Both floods and floodwall failures are assumed to be binary events; thus floodwall will either hold or fail completely, resulting in either no damage or complete damage of all assets in the floodplain. Additionally, the analysis will be conducted assuming constant conditions over the 30 year period and not consider non-stationary conditions or discount rates. Finally, the comparison will only evaluate damage and construction costs, and will not consider other impacts such as lives lost or secondary economic losses.

There are four uncertain factors that impact total costs. The first uncertain factor is the flood return period. Historical data suggest a flood return period of 100 years, but climate change could alter precipitation amounts for the region, in turn impacting the frequency of flooding. Climate model projections of future precipitation in the region differ, with some models predicting an increase in rainfall while others predict a decrease. Because of this, there is significant uncertainty about what the flood return period will be in the future. The second uncertain factor is the failure probability of the existing floodwall. The floodwall was installed many years ago and city engineers disagree about its failure probability should a flood occur. However, if the upgrades are installed, they will result in a floodwall reliability of $99 \%$; thus, only the reliability of the existing floodwall is uncertain. The third uncertain factor is the value of assets that will be present in the floodplain in the future (including the costs to repair the floodwall should it fail), as this depends on the rate of development in the city in years to come. The fourth uncertain factor is construction costs. While the city has obtained preliminary cost estimates from 
multiple contractors, there is disagreement about how much upgrades are likely to cost. For simplicity, these four uncertain quantities are all assumed to be independent of each other.

It is important to recognize that each of these four quantities (flood return period, floodwall failure probability, assets at risk, and construction costs) are all epistemically uncertain. They are not inherently variable or repeatable events; thus any probabilistic description of their values represents a Bayesian degree of belief. However, two quantities (the flood return period and number of floodwall failures) are parameters that describe frequentist models of varying phenomena (Figure 2). For a given flood return period, the distribution of the number of floods that will occur over the 30-year period of analysis is modeled using a Poisson distribution. Given a floodwall failure probability and number of floods occurring, the number of floodwall failures that will occur over the 30 -year period is modeled as a binomial distribution. Based on this, the total costs are modeled as:

$$
\text { Equation 1: } \quad C_{\text {Total }}=C_{C}+C_{D}=C_{C}+A * N_{\text {fail }}
$$

where $C_{\text {total }}, C_{C}$ and $C_{D}$ are the total, construction, and damage costs, respectively, $A$ is assets at risk, and $N_{\text {fail }}$ is the number of floodwall failures that occur over the 30-year period of analysis. Note that the number of floodwall failures cannot exceed the number of floods that occur. If the upgrades are not installed, then construction costs are equal to zero. We assume all dollar values are in present-year dollars.

\section{METHODOLOGICAL COMPARISON}

\subsection{Probabilistic Analysis with Uncertainty Factors}

Semi-quantitative uncertainty factors have been proposed as an additional component of risk assessment aimed at communicating the level of underlying knowledge supporting the assessment, thus providing a more "comprehensive risk picture" than expected values and probabilities alone ${ }^{(4)}$. The term "uncertainty factors" refers to limitations in background knowledge that can be hidden in the assumptions made to conduct the assessment. This method aims to identify these assumptions and assess the strength of knowledge on which they are based, as well as the degree to which their violation would impact the quantitative results of the assessment (sensitivity). For example, an assumption has strong supporting knowledge if the involved phenomena are well understood and there is reliable data and expert consensus supporting the assumption ${ }^{(27)}$. An assumption has a high sensitivity if its violation results in dramatic changes to the quantitative results of the risk assessment.

For this approach, each of the four uncertain input quantities can be described probabilistically based on expert elicitation. However, because the flood return period and floodwall failure probability are parameters used to describe inherently variable phenomenon, allowing for uncertainty in these quantities 
would result in multiple distributions of the frequency of floods and floodwall failures. While this corresponds to the "probability of frequency" analysis described by Kaplan and Garrick ${ }^{(11)}$ and the highest level of treatment of uncertainty outlined by Pate-Cornell ${ }^{(2)}$, we avoid this treatment to highlight the role of uncertainty factors and be consistent with typical risk assessment practices. Table I shows how each of the four uncertain input quantities are represented, along with key assumptions on which those representations rest:

- The flood return period is estimated to be 50 years. This is based on the assumption that average annual precipitation in the region will increase by $15 \%$ relative to historic levels. A precipitation increase of $15 \%$ is the average projection from multiple climate models.

- Multiple engineers have inspected the existing floodwall, and their elicited judgments on the probability of failure range from 0.1 to 0.2 . The probabilistic assessment assumes a failure probability of 0.15 , equal to the central value.

- The value of assets at risk in the floodplain depends on economic development and population growth. Based on ranges of growth experienced in the past, the value of assets that will be present in the future is represented by a uniform distribution from $\$ 500 \mathrm{M}$ to $\$ 600 \mathrm{M}$. This assessment is based on the assumption that growth rates will not exceed those experienced historically.

- A distribution of possible costs is elicited from the lead city engineer based on preliminary cost estimates and their previous experience with similar projects. Their beliefs are found to roughly approximate a lognormal distribution with a mean of $\$ 45 \mathrm{M}$ and standard deviation of $\$ 7 \mathrm{M}$, so this distribution is used for sampling and carrying out the probabilistic analysis. However, this distribution assumes that prices for land and materials remain consistent with current levels.

These quantities are used to determine a distribution of total costs for the existing and upgraded floodwall using 10,000-fold paired Monte Carlo simulations. The distribution of costs for each alternative is shown in Figure 3, and summary statistics are shown in Table II. The expected total costs are similar for both alternatives ( $\$ 53.08 \mathrm{M}$ and $\$ 47.99 \mathrm{M}$ for no-action and upgrades, respectively). However, the full distributions of cost for the two alternatives differ substantially. If no-action is taken, the probability of incurring no costs is 0.91 , with a 0.09 probability of incurring costs over $\$ 500 \mathrm{M}$. If the upgrades are constructed, then there is a 95\% probability that the costs will be between $\$ 32.85 \mathrm{M}$ and $\$ 61.32 \mathrm{M}$.

Table III shows the qualitative assessment of each of the assumptions used to probabilistically represent the uncertain input quantities. The strength of knowledge supporting each assumption is the same regardless of which alternative is being evaluated, but the sensitivity of each assumption (i.e., the 
degree to which the cost associated with each alternative could vary should an assumption be violated) can vary between alternatives. For example, the distribution of costs for upgrades relies on the assumption that land and material costs remain consistent with current levels, but the distribution of costs for the noaction alternative does not rely on this assumption.

The assumption that rainfall increases by $15 \%$ is based on the average projection of changing precipitation from multiple climate models. This means that some models project increases greater than $15 \%$ and some models project increases less than $15 \%$ or perhaps even decreases. There is no reason to believe that this average is more likely than any single model's projection, nor that the models evaluated capture the complete range that could occur. Thus, it is entirely possible that the change in rainfall could be something other than $15 \%$. Therefore, the strength of knowledge supporting this assumption is judged to be weak. The assumption regarding failure probability is assigned a moderate strength of knowledge. While the majority of engineers believe the failure probability to be close to the central value, the possibility for more extreme values cannot be ruled out. The assumption about asset growth rates is assigned a high strength of knowledge since extensive long-term historical records exist on growth rates in the city and include periods of very rapid and even negative growth. Finally, the assumption about land and material costs is assigned a weak level of knowledge, since these prices have historically fluctuated and there is no reason to believe that they would not do so in the future.

The no-action alternative is judged to be moderately sensitive to the assumptions regarding rainfall, floodwall failure probability, and asset growth rates. The violation of these assumptions could change the probability of incurring a certain level of damage costs, but does not automatically result in damage costs since the number of floods and floodwall failures are subject to aleatory uncertainty. The upgrades alternative is judged to have a low sensitivity to the rainfall and assets assumptions, since the low failure probability of the upgraded floodwall means that even if more floods occur and assets at risk are high, it is still unlikely that damage will occur. The upgrades alternative is not impacted by the assumption regarding the existing floodwall failure probability, just as the no-action alternative is not impacted by the assumption about land and material costs. However, the upgrades alternative is highly sensitive to the assumption about land and material costs, since construction costs are the main contributor to total costs for this alternative and could be significantly higher if this assumption is violated.

These qualitative assessments can be combined into an overall judgment on the strength of knowledge supporting the probabilistic analysis as a whole. For the no-action alternative, the three assumptions that could impact total costs vary in terms of strength of knowledge but could all have a moderate impact on the quantitative results. Thus, the strength of knowledge supporting the evaluation of 
1 the no-action alternative is judged to be moderate. The evaluation of the upgrades alternative is also assigned a moderate strength of knowledge since there is only one assumption that could greatly impact it but the strength of knowledge supporting that assumption is low. It should be noted that the "strength of knowledge" is a judgement evaluation, and thus a subjective reflection of the analysts' beliefs and not "uncertain" in the same sense that model parameters and input quantities are. That said, as a subjective judgement it is certainly possible that two people regarding the same underlying knowledge that supports a probabilistic assessment (i.e., amount and quality of data, understanding of the phenomena studied, model accuracy, etc.) may come to different conclusions about whether that knowledge should be considered strong, moderate, or weak. Ideally, the scores should be presented along with some rationale for why that score was chosen, so that the limitations in data, model representation, expert consensus, etc. are made clear. This not only would provide a more comprehensive description of the risk, but would also help identify places where strength of knowledge could be improved (e.g., through additional evaluation or model refinement) and those areas where that may not be possible (perhaps due to strong disagreement between experts).

\subsection{Probability Bounds Analysis}

Probability bounds analysis (PBA) has been proposed as method to distinguish between aleatory and epistemic uncertainty ${ }^{(13)}$. This method employs imprecise probability distributions to describe uncertain parameters, which in the simplest case can arise from aleatory uncertainty being represented using probability distributions, and epistemic uncertainty being represented using intervals. These two forms of uncertainty can be combined and propagated using standard mathematical procedures, resulting in bounds on a cumulative distribution function, referred to as p-boxes.

In the example problem, the flood return period, existing floodwall failure probability, and the assets at risk are all epistemically uncertain, and thus represented as intervals (Table IV). The representation of these quantities in such a matter allows us to relax some of the assumptions that were required in the fully probabilistic analysis. For example, the probabilistic analysis assumed that annual rainfall would increase by $15 \%$ in the future, which was the average projection from multiple models whose individual projections ranged from decreases of $5 \%$ to increases of $25 \%$. Instead of assuming that actual rainfall change will be equivalent to this model average, we can represent the flood return period as a range (20 years in the case of rainfall increase of $25 \%$ to 200 years in the case of a rainfall decrease of $5 \%$ ). However, it is important to recognize that this analysis is still based on other (albeit weaker) assumptions. In the case of the flood return period, we still assume that the actual change in rainfall is captured within the model range. 
In some cases, an uncertain quantity could be representative of both epistemic and aleatory uncertainty. For instance, the construction costs could be a function of two uncertain values: the total quantity of materials required, and the unit costs for these materials. The total quantity of materials required is epistemically uncertain, whereas the unit costs for materials may fluctuate through time and thus exhibit aleatory variability. Explicitly representing each of these quantities in the PBA framework would require combination of an interval encompassing possible material quantities and a probability distribution representing unit costs. Combining these two parameters would result in an imprecise probability distribution, as shown in Figure 4. One can envision other situations where an imprecise probabilistic distribution for construction costs could arise as well. For example, an expert may be uncomfortable assigning precise values during probability elicitation procedures and prefer to express his degree of belief as ranges of probabilities, or elicitations may have been conducted on multiple experts, resulting in multiple probability distributions. In this paper we do not quantitatively explore all of the situations that could result in this imprecise distribution, but will instead simply assume that possible distributions of construction costs are found to approximate an imprecise lognormal distribution with a

understanding the rationale for imprecise representation of this quantity will be important when interpreting the final results of the analysis, and will be revisited in subsequent sections.

Probability bounds for the two alternatives were computed using the Williamson and Downs (28) algorithm as described by Tucker and Ferson ${ }^{(29)}$. Instead of point values for summary measures such as expected value, we now have ranges for each of these values (Table V). Whereas the expected values for each alternative were fairly close in the probabilistic assessment, the range in expected value for the noaction alternative is much wider than the range for the upgrades. The interpretation of this range depends on the rationale for using imprecise probabilities. In the case of the no-action alternative, the total costs are a function of three uncertain quantities: the flood return period, floodwall failure probability, and assets at risk. Because each of these are epistemically uncertain, they were represented as intervals, while variability in the number of floods and floodwall failures that will occur is modeled using probability distributions. In the resulting p-box for total costs, epistemic uncertainty regarding the three input quantities contributes to the width of the box, while aleatory uncertainty in the number of floods and floodwall failures contributes to the tilt. This is consistent with Ferson and Ginzburg's ${ }^{(13)}$ interpretation of uncertainty representation in PBA. Similarly, the range for expected value can be interpreted as resulting from lack of knowledge regarding the flood return period, floodwall failure probability, and assets at risk. 
The interpretation for the total cost of upgrades is different because this depends on construction costs, as well as the flood return period and assets at risk. In this case, the construction costs are characterized by epistemic uncertainty in the amount of materials required and aleatory uncertainty regarding future material costs. Therefore, the range of expected values can be interpreted as resulting from lack of knowledge regarding the flood return period, assets at risk, and the quantity of materials required. However, if probability bounds were used for other reasons, such as imprecision in elicited probabilities, this range would result from epistemic uncertainty regarding the flood return period and assets at risk, as well imprecision in the expert's elicited beliefs regarding construction costs. Similarly, if the bounds on the distribution of construction costs resulted eliciting probabilities from multiple experts, the range in expected values would partly result from disagreement in expert's elicited beliefs.

\subsection{Robust Decision Making}

Robust decision making ${ }^{(15)}$ is a multi-step, iterative process aimed at identifying and designing robust strategies, where robustness implies satisfactory performance in conditions other than those for which the system was designed. The process consists of both quantitative analysis and qualitative deliberation and review. In this comparison, we evaluate one of the key analytical components of the process, referred to as "scenario discovery." This process is motivated by the assumption that in highly uncertain situations, it is unlikely that any single alternative will be robust to all conditions it might encounter. For example, in our flood risk problem, the no-action alternative could result in large damage costs if the flood return period is low and the floodwall failure probability is high. However, the upgrades are likely to result in unnecessary construction costs if the flood return period is very high. Therefore, the scenario discovery process aims to identify regions in the input variable space that result in undesirable outcomes. These regions are used to create a quantitative description of scenarios where an alternative will fail to meet its goals ${ }^{(30)}$.

The scenario discovery process runs hundreds to thousands of simulations to assess system performance under different combinations of input variables. The patient rule induction method (PRIM) bump-hunting algorithm ${ }^{(31)}$ is then applied to the simulation results. The objective of the PRIM algorithm is to find regions of the input variable space $\boldsymbol{X}$ that result in particularly high values of the output variable $Y=f(\boldsymbol{X})$. This region $R$ is made up of one or more "boxes" $B$ that can be defined by simple logical conditions involving the value of individual input variables ${ }^{(31)}$. In our flood risk example, the input variables $\boldsymbol{X}$ include the flood return period, floodwall failure probability, assets at risk, and construction costs. We could thus define an example box $B_{k}$ as the set of simulations containing input variables defined by a flood return period below 100 years and assets at risk greater than $\$ 580 \mathrm{M}$. 
To identify these boxes, the algorithm uses top-down successive refinement, referred to as "peeling," followed by bottom-up successive expansion ("pasting"). The peeling phase begins with a box

$B_{0}$ containing all of the data. At each iteration, a small sub-box $b^{*}$ is removed, resulting in a smaller box equal to $B-b^{*}$. The sub-box $b^{*}$ chosen for removal is selected from a set of candidate sub-boxes, each of which is defined by a single input variable $x_{j}$, to maximize the mean value of $Y$ within the resulting box $B$ $b^{*}{ }^{\left({ }^{11}\right)}$. This process is continued until the size of the box falls below a pre-specified value. The pasting process then readjusts the boundaries of this box by essentially reversing the peeling algorithm. In this stage, a small box $b^{*}$ is added to the existing box $B$ from a set of candidate sub-boxes to maximize the mean value of $Y$ in the new larger box $B+b^{*}$. This process continues until the mean of the larger boxes starts to decrease. The peeling and pasting algorithm can be repeated on remaining subsets of the data to obtain a set of boxes that collectively include a sufficiently high portion of the input space where the output $Y$ assumes large values ${ }^{(31)}$.

For the flood risk example, we apply scenario discovery to identify the conditions where the upgrades result in lower costs than the no-action alternative. Because the objective of running the simulations is not to develop a distribution of total costs but instead to create diverse combinations of input quantities, 10,000 Latin hypercube samples were created using relatively wide distributions for each of the four uncertain input quantities (Table VI). The flood return period, floodwall failure probability, and assets at risk were all sampled from uniform distributions over the intervals used in the probability bounds analysis. The construction costs were sampled from a lognormal distribution with a mean of $\$ 45 \mathrm{M}$ (the same value use in the probabilistic analysis) and standard deviation of $\$ 10$ (the largest standard deviation used in the probability bounds analysis).

No action results in lower total costs than conducting the upgrades in $95 \%$ of the simulations. However, this result should not be interpreted as a probabilistic statement since we make no assumptions that each simulation is equally likely. There were 532 simulations where the upgrades had lower total costs than no-action. The SDtoolkit package in $\mathrm{R}^{(32)}$ was used to run the PRIM algorithm on the simulation results and identify boxes that describe the 532 simulations where repairs resulted in lower cost than no-action (for brevity, we refer to these as "high-cost simulations"). The first step of the process creates a plot called a "peeling trajectory" which shows the sequence of boxes created through the peeling process described above (Figure 6). Coverage refers to the percentage of high-cost simulations captured by a box, while density refers to the percentage of simulations in the box that are high-cost. The peeling process starts at the lower right hand corner of the figure, with a box (represented by an unfilled circle) that includes all of the simulations. In this case, the coverage is 1.0 (it contains all of the high-cost simulations) but the density is only 0.05 (since only $5 \%$ of these simulations were high-cost). As the 
1 peeling process continues from right to left, the boxes become progressively smaller, resulting in decreases in coverage but increases in density. The number of restricted variables also increases as the peeling process continues, so that the boxes identified earlier in the algorithm are only defined by one variable, while the boxes identified later on are defined by multiple variables.

Through visual inspection of this figure, it is evident that the majority of boxes have a very low density. For example, the circular 1-dimensional box at the far right hand end of the plot represents all simulations where the flood return period was less than 182 years. This box's coverage is $100 \%$ but its density is only $5.3 \%$; that is, it describes $100 \%$ of the high-cost simulations, but only $5.3 \%$ of the simulations with a flood return period of less than 182 years result in high-costs. Moving from right to left along the curve results in increasingly complex boxes with sizable decreases in coverage but only small increases in density. This result makes intuitive sense when one considers that there is a considerable amount of random variation that determines whether or not damage costs are incurred. For example, one could consider a "worst-case" scenario for the no-action alternative where the flood return period is low, the floodwall failure probability is high, and the assets at risk are high. Even under this scenario it is still subject to aleatory variation.

The input variables used to define the boxes can be informative. For example, the circular onedimensional boxes are all described by the flood return period. The diamond-shaped two-dimensional boxes are described by flood return period and floodwall failure probability. Because the objective of the PRIM algorithm is to identify combinations of input quantities that most effectively predict whether the no-action alternative will result in higher costs than the upgrades, this provides an indication of which uncertain input quantities drive the decision. Ultimately, a user would select a box, or combination of boxes, that provides a satisfactory balance between coverage, density, and interpretability. This choice is of course subjective, and it is possible (particularly in a case like our example) that none of the boxes will be deemed sufficiently informative. For discussion purposes, we select the two-dimensional box with the greatest coverage: this box includes all simulations where the flood return period is less than 83 years and the floodwall failure probability is greater than 0.11 . It has a coverage of $60 \%$, but a density of only $9 \%$. We could then infer that there are two scenarios that would drive our decision about whether or not the upgrades should be installed. The first scenario would be that the flood return period is less than 83 years and the failure probability is greater than 0.11 , and the second scenario would be if these conditions were not met. One could envision this informing a subsequent probabilistic analysis, where instead of developing probabilistic (or probability-bounds) distributions for the entire range of outcomes for each 
uncertain input quantity, expert elicitation would focus only on the relative likelihood of those two scenarios.

\section{COMPARISON RESULTS}

The above sections describe how three diverse methods can be applied to the same simple example problem related to flood risk under deep uncertainty. In this section, we compare the approaches in terms of their representation of uncertain quantities, their analytical output and its interpretation, and their implications for practical decision making. A summary of this comparison is presented in Table VII.

\subsection{Representation of uncertain quantities}

Each methodology takes a different approach in the manner in which it represents uncertain quantities. In the uncertainty factors analysis, all uncertain quantities were represented using first order precise probabilistic distributions. However, second order probability models could also be used if probability distributions were assigned to represent the analysts' beliefs regarding the flood return period and floodwall failure probability. In general, the approach can accommodate one or two probability levels so long as frequentist models based on repeatable events can be justified to represent aleatory variation, as discussed by Aven ${ }^{(26)}$. In either case, the method focuses less on exact quantitative description of all uncertain quantities that may impact assessment results, and more on identifying underlying assumptions on which probabilistic descriptions of uncertainty (whether they represent the analysts' beliefs or repeatable phenomena) are based.

The probability bounds analysis represents uncertain parameters using imprecise probabilistic distributions. In the simplest case, these imprecise distribution result from treating quantities that are epistemically uncertain as intervals and treating quantities that are inherently variable as probability distributions, although they can also arise from other circumstances as described by Tucker and Ferson ${ }^{(29)}$. This has the advantage of allowing for imprecision in probabilistic judgements when underlying data to support precise distributions may be limited. However, it has been pointed out that in cases where there is little data or underlying knowledge to describe a probabilistic model for aleatory phenomenon (such as the number of floods in a 30 year period) there may be even less basis to describe upper and lower bounds on the parameters that describe that phenomena (such as the flood return period) ${ }^{(33)}$. Thus, even the use of intervals to describe epistemically uncertain quantities may be questioned in situations where appropriate bounds on those quantities is subject to disagreement or uncertainty.

In the RDM analysis, uncertain quantities can be represented as intervals (which are effectively treated as uniform distributions for sampling purposes in accordance with the principle of indifference ${ }^{(34)}$ and entropy maximization ${ }^{(35)}$ ) or probabilistic distributions, and the method does not distinguish between epistemic and aleatory uncertainty. Because our example was focused on the scenario discovery process 
of identifying scenarios that result in poor outcomes for an alternative, uncertain quantities in our example were represented using relatively non-informative probabilistic distributions that were used to generate samples across the uncertainty space. However, it is important to remember that these samples should not be interpreted in a probabilistic manner. Instead, each sample is interpreted as a "plausible future states of the world" ${ }^{\text {(15) }}$ which could conceivably occur, but does not assume that each sample is equally likely as would be the case in a Monte Carlo simulation.

These different representations of uncertainty shed some light on the types of situations where one approach might be more suitable than the others. The uncertainty factors approach represents uncertain parameters in the same manner they are represented in traditional PRA, and is likely to be appealing in situations where decision makers would like to conduct a probabilistic assessment but with a more comprehensive understanding of the underlying knowledge on which that assessment is based. The manner in which probability bounds analysis can provide a measure of discernment between aleatory variability and epistemic uncertainty may be valuable in contexts where the underlying phenomena can be meaningfully separated into these two categories and where decisions makers are concerned about what degree of risk stems from reducible versus non-reducible uncertainties. However, it is important to keep in mind that this separation is only meaningful if the use of frequentist probability models based on repeatable events can be justified to describe aleatory uncertainty ${ }^{(26)}$. Furthermore, this method may become contentious in situations where there are not obvious upper and lower bounds to describe epistemic uncertainties. Finally, while the RDM methodology can use probabilistic distributions to generate samples across the uncertainty space, its analytical focus is not on determining the probability of different outcomes but instead on understanding the conditions where one alternative might be preferred over the other. This focus may be valuable when there is strong disagreement regarding the distribution of uncertain parameters or the likelihood of different conditions which are unlikely to be resolved through the use of imprecise probabilities.

\subsection{Analytical output}

The analytical output for the uncertainty factors assessment includes both the quantitative description of the distribution of total costs associated with each alternative, as well as the qualitative description of the underlying knowledge supporting this assessment. The quantitative output indicates that the expected costs between the two alternatives are relatively comparable, with the no-action and upgrades alternatives having expected costs of $\$ 53.08 \mathrm{M}$ and $\$ 47.99 \mathrm{M}$, respectively. However, the distribution of costs for the no-action alternative is much wider than the upgrades, with a 95\% uncertainty interval of $\$ 0$ to $\$ 576 \mathrm{M}$, compared to an interval of $\$ 32.9 \mathrm{M}$ to $\$ 61.3 \mathrm{M}$ for the upgrades. The strength of knowledge supporting these judgments is considered to be moderate for each alternative, suggesting that 
the phenomena may be well understood but modeled in a simple or crude way, or that only some reliable data to characterize the phenomena are available ${ }^{(27)}$. Explicit description of the impact that each assumption could have on the different alternatives could be particularly useful when the strength of knowledge supporting the assessment of one alternative is significantly higher than the other, since this would highlight those actions that might be relatively "sure bets" (i.e., actions that are well understood and supported by extensive experience and data) and those whose impacts on risk are less well understood.. The systematic evaluation of each individual assumption also helps illuminate which assumptions are most critical to the validity of the quantitative assessment. Because it has a weak strength of knowledge and a moderate impact on no-action costs, the assumption regarding future rainfall levels appears most critical to the quantitative assessment of the no-action alternative. Meanwhile, the assumption regarding land and material costs is most critical to the upgrade alternative, because it has a weak strength of knowledge and a high impact on upgrade costs. This systematic evaluation and presentation of underlying assumptions highlights the limitations of the risk assessment, areas where it could potentially be improved, and areas where surprises related to conditions that deviate from the assessment's assumptions could be most consequential.

In the probability bounds analysis, the probabilistic distributions of costs from each alternative are now represented by imprecise p-boxes, and probabilistic summary statistics are represented by an interval of possible values rather than a precise value. The expected costs associated with the no-action alternative is represented by the interval from $\$ 7 \mathrm{M}$ to $\$ 182 \mathrm{M}$, while the expected costs associated with the upgrades are represented by the interval from $\$ 37.12 \mathrm{M}$ to $\$ 63.95 \mathrm{M}$. From this description, it is apparent that the expected cost of the upgrades may be higher or lower than the expected cost for no-action, but that the noaction alternative has the potential for a much higher expected cost than the upgrades. Visual examination of the p-boxes for each alternative can provide an understanding of the relative contribution of epistemic and aleatory uncertainty associated with each alternative as well. In comparing the two p-boxes, it is apparent that the total costs associated with the upgrades are associated with both less aleatory uncertainty (represented by a steeper cumulative distribution function) and less epistemic uncertainty (represented by the thickness of the p-box). While this can provide some insight into the cumulative effect of epistemic uncertainty on assessment outcomes, it does not provide any information about the relative contribution of each input quantity. Thus, the decision maker may find that the resulting p-boxes are unsatisfactorily wide, but have no insights into which uncertainties have the greatest impact on this width and thus possible ways for additional research to reduce this width. Obtaining this information would require additional analysis, for example through a sensitivity analysis as outlined by Ferson and Tucker ${ }^{(36)}$. 
The analytical output from the RDM assessment takes a very different form. The results do not describe the likelihood of all outcomes that may occur, under the assumption that the limited knowledge

available to support such an assessment would undermine its validity ${ }^{(15)}$. Instead, it provides an evaluation of the conditions where the upgrades might be preferred over the no-action alternative, in the form of a scenario defined by a flood return period below 83 years and a floodwall failure probability greater than 0.11. While this is not as informative as a full distribution of the likelihood of different outcomes, this serves to reduce the complexity of a given problem to something more understandable. It would be possible to combine this approach with a later probabilistic analysis where the relative likelihood of different scenarios are evaluated, providing information more in line with that of a traditional risk assessment. This also provides insights into the uncertain inputs that have the most influence on the relative performance of the two alternatives, suggesting areas of additional research that may be particularly valuable. Additionally, the simulation process identified that the no-action alternative resulted in lower costs in the majority of simulations (95\%) but this output cannot be interpreted in a probabilistic manner unless each simulation can assumed to be equally likely.

In both the uncertainty factors and RDM analyses, a significant portion of the analytical output contributes towards a better understanding of which assumptions and uncertain quantities have the greatest impact on risk characterization and the choice between alternatives. This information can be particularly useful within the context of adaptive risk management, which provides a framework for managing risks in situations when system response to different management alternatives cannot be confidently predicted ${ }^{(37)}$. Understanding the areas where a reduction in uncertainty or additional refinement of the risk assessment would be most valuable can help prioritize areas for additional research or monitoring, providing opportunities for learning that can be incorporated into a continuing process of ongoing risk management. Concerns over deep uncertainty have prompted the development of a number of frameworks for learning and prioritization of alternatives under deep uncertainty. For instance, resilience analytics ${ }^{(17,18,38)}$ provides a methodology for identifying which uncertain future scenarios have the greatest potential to disrupt risk management priorities, allowing decision makers to focus their attention on uncertainties that matter most to their particular system. However, one difference with this approach is that it still assesses scenarios that are defined beforehand rather than identifying them through analysis as is done in RDM. Additional research on these different methods for learning within adaptive risk management frameworks could provide valuable insights into managing risks under deep uncertainty.

\subsection{Implications for risk management and decision making}

It has been acknowledged that risk assessment, particularly in cases of deep uncertainty, is conducted within a context of deliberative review and managerial judgment ${ }^{(39)}$, and that decisions related 
to the risk in question should be considered "risk-informed", rather than "risk-based" (40). This is particularly true in cases of highly uncertain or ambiguous risk problems, which require a management approaches based on precaution and discourse that are aimed at producing collective understanding and identifying mutually acceptable solutions ${ }^{(41)}$. For this reason, it is useful to consider how each approach contributes to improving understanding of the risks in a manner that could support and inform decisionmaking. However, it is important to recognize that none of the assessed methods are purely prescriptive; instead of identifying "an optimal decision", they instead aim to characterize risk and uncertainty in a way that can inform decision making. While each methodology could be used in a prescriptive manner when certain decision rules are assumed, these decisions rules are likely to be contentious in deeply uncertain conditions, particularly when multiple viewpoints and values must be considered. While these conditions make objectively "correct" risk management solutions elusive, risk assessment can inform decision making in other ways; for example, by identifying which aspects of a system contribute most significantly to risk and which measures offer the greatest risk reduction potential ${ }^{(42)}$. For this reason, we avoid a prescriptive interpretation of analytical results here and instead focus on the insights that each approach would provide to support managerial review and judgement.

In the uncertainty factors assessment, the expected value of the upgrades is slightly lower than the no-action alternative, but has a much smaller probability of incurring very high costs. This would suggest to many decision makers that the upgrades are a worthwhile investment, but the caveat that the strength of knowledge supporting this assessment is only deemed to be moderate might make planners less confident in that decision. However, one useful insight to arise from the qualitative component of the assessment is the identification of which assumptions are most critical for the quantitative risk assessment, as this can point towards assumptions that should be given additional consideration through either further research or more sophisticated treatment within the risk assessment. For instance, because the assumptions regarding future rainfall rates and land and material costs appear to be the most critical for comparison of the two alternatives, that might suggest that additional research aimed at understanding the validity of those assumptions would be a useful course of action. By providing improved knowledge and clarification about the risk context, this insight could be used to support an ongoing process of adaptive risk management ${ }^{(43)}$. Alternatively, the risk assessment process could be repeated, but with these assumptions relaxed through the use of intervals or imprecise distributions to describe the flood return period and land and material costs, as suggested by Berner and Flage ${ }^{(33)}$. This ability to refine the risk assessment or focus research activities could be one reason that including information on the background information that supports quantitative risk descriptions improves the perceived usefulness of risk assessments ${ }^{(44)}$. However, one limitation with this approach may be the timing of the assessment. Ideally, if the strength of 
knowledge supporting an assessment is low, this could be identified and communicated prior to conducting a possibly resource-intensive quantitative assessment.

In the probability bounds assessment, it becomes apparent that epistemic uncertainty makes it unclear which of the two alternatives actually has a lower expected costs. However, the probability of very high costs (for instance, above $\$ 500 \mathrm{M}$ ) could be as high as 0.8 for the no-action alternative. It should be noted that one common critique of alternative uncertainty representations is that they might result in bounds that are too wide to support decision-making ${ }^{(45)}$, but this example demonstrates that this will not always be the case. While the bounds for the no-action alternative in our example are quite wide, this demonstrates that the impact of epistemic uncertainty on the no-action alternative appears much larger than the impact of epistemic uncertainty on the upgrades. This suggests that the possible costs associated with the upgrades are well characterized compared to the costs of doing nothing, and puts the upgrades in a relatively favorable light. The potential for very high costs associated with the no-action alternative due to both epistemic and aleatory uncertainty would likely suggest to decision makers that the upgrades would be a worthwhile investment.

In the RDM assessment, the analytical output provides more insight regarding the key uncertainties driving the decision, and thus the most valuable areas for further research and evaluation, rather than the comparison between the two alternatives. In this case, the key uncertainties were the flood return period and the floodwall failure probability. In particular, the no-action alternative appears particularly vulnerable to a scenario where the flood return period is less than 83 years and the floodwall failure probability is greater than 0.11 . This would suggest that decision makers should base their decision around the likelihood of these specific conditions, rather than worrying about the full distribution of all uncertain parameters. If there is strong disagreement or uncertainty surrounding the likelihood of this scenario, it would suggest that additional research in this area would be valuable. The analysis also found that the no-action alternative resulted in lower costs in $95 \%$ of simulations, which at first glance might make the upgrades appear unnecessary. However, one must exercise caution in basing decisions on these results since they do not consider the likelihood or magnitude of costs in each case. While the magnitude of costs could be evaluated by using alternative performance measures based on regret-based or deviationbased metrics as in Lempert et al. ${ }^{(15)}$ and Kasprzyk et al. ${ }^{(46)}$, these measures are still reliant on the assumption that each simulation is equally likely. If there is strong disagreement surrounding the distributions used to generate the samples, this assumption is likely to be contentious.

It is interesting to note that the implications for risk management (both in terms of the choice between alternatives and suggested areas for additional research or refinement of the risk assessment process) are not necessarily consistent between the three approaches. The uncertainty factors assessment 
and probability bounds analysis both made the upgrades alternative appear quite favorable, while the RDM assessment (and particularly the fact that the majority of simulations resulted in higher costs for the upgrades) could suggest to some decision makers that these upgrades are not necessary. While the probability bounds analysis suggested that the costs associated with the no-action alternative were much more sensitive to epistemic uncertainty than the upgrades, the uncertainty factors assessment suggested that the strength of knowledge supporting the assessment of each alternative was equal. This is likely due to the fact that the upgrade costs were judged to be highly sensitive to the assumption regarding land and material costs, which had weak supporting knowledge. While a violation of this assumption may result in high upgrade costs compared to the baseline quantitative risk assessment, these costs may still be quite small compared to the distribution of costs for the no-action alternative. Finally, the RDM assessment suggested that land and material costs were not actually a very important parameter in choosing between the two alternatives, and instead suggested that the flood return period and floodwall failure probability were most important. The divergence between risk management implications that occurred even within this very simple example suggest that further research comparing these methodologies using a more realistic problem could be very valuable.

\section{DISCUSSION}

Relating each process back to the risk assessment framework described in Section 2 allows us to clarify how each methodology contributes to this risk assessment process and resulting risk description. Uncertainty factors were specifically developed to clearly demonstrate the underlying strength of knowledge for a risk assessment, and thus provide each of the components of the risk description used here. In our example, $A$ ' and $C$ ' refer to a certain number of failures, combined with a specific realization of asset value and construction cost, and the total costs that result. The uncertainty associated with each of these realizations is measured probabilistically, and $K$ is the qualitative description of the underlying knowledge on which these probability measurements are based.

The PBA provides the same description of $A^{\prime}$ and $C^{\prime}$, but the uncertainty measurement $Q$ is now the bounds on the probability associated with $A$ ' and $C$ '. The underlying knowledge $K$ is not explicitly described, but could be inferred by the shape of the resulting p-box for total costs in each case. This is a frequent interpretation of p-boxes, where the slant of the box represents variability while the width represents epistemic uncertainty. However, this interpretation depends on what led to the use of imprecise probability distributions in the first place. As discussed in Section 3, the width of the p-box for total costs of the no-action alternative can be interpreted as the epistemic uncertainty associated with this alternative, since the imprecision in this distribution resulted entirely from lack of knowledge regarding the flood return period, floodwall failure probability, and assets at risk. However, the width of the p-box for the 
repairs alternative could arise from multiple sources, including epistemic uncertainty, imprecision in elicited probabilities, or disagreement between experts. Thus, the precise interpretation of the PBA results requires careful consideration of the various sources of uncertainty and imprecision leading to that result.

The RDM analysis is the most challenging to relate back to the risk assessment process and description, but could be interpreted in one of two ways. The first could be that it serves as an alternative method for hazard identification which identifies specific combinations of uncertain conditions that lead to (or are relatively likely to lead to) undesirable outcomes. Another interpretation could be that it provides an alternative description of $A^{\prime}$ and $C^{\prime}$, where instead of considering any possible outcome $A^{\prime}$ and its consequences $C$ ', an "event" is simply a box that results in particularly high consequences $C$ '. In this interpretation, the set of outcomes $A$ ' includes only Scenario 1 (flood return period is less than 83 years and the failure probability is greater than 0.11 ) and Scenario 2 (the above conditions are not met), rather than all possible outcomes. This interpretation could be useful in situations where decision-makers are overwhelmed by having to consider all outcomes that could occur, and prefer to consider a smaller number of scenarios that would drive their decision. However, one limitation with this interpretation is that there is not a clear set of consequences associated with each of those two scenarios due to the presence of aleatory uncertainty in the number of floods and floodwall failures that will occur. Additionally, it provides no measurement of the relative likelihood or uncertainty associated these events $\left(Q^{\prime}\right)$, and thus no description of the underlying knowledge $K$ for that measurement.

One advantage of the uncertainty factors methodology is its explicit description of the assumptions underlying the analysis, as well as the likelihood and consequences of their violation. While explicit description of underlying assumptions should be a component of any risk assessment, this may not always be the case. By creating an explicit framework for describing these assumptions and their impacts, uncertainty factors could make presentation of these assumptions more systematic and effective. While the assumptions underlying the probability bounds and RDM analyses are relaxed relative to the probabilistic analysis, they will always be present since complete modeling of all uncertainties and complexities is impossible in all but the most trivial of systems. Developing systematic and transparent methods for description and assessment of underlying assumptions for different technical approaches could be a valuable area of research for any method of risk and uncertainty assessment, and could lead to important steps forward in improving the transparency of these analyses.

\section{CONCLUSIONS}

In this paper, we compare three methods that have been proposed for risk assessment under deep uncertainty and critically evaluate how these approaches contribute to the risk assessment process and resulting risk description. By applying each method to a stylized example problem related to flood risks 
under climate change, we are able to compare each approach's representation of uncertain quantities, analytical output, and implications for risk management. While each methodology aims to assess and describe risks in a manner that is more reflective of the uncertainties and assumptions underlying the assessment, the analytical output and implications for decision making are not necessarily consistent between approaches. This suggests the potential value in additional comparative research to better understand the sources of these deviations, as well as the need for analysts to consider the ways in which the choice of methodology might impact analytical results. Additionally, differences in the representation of uncertain quantities and analytical output associated with each approach suggest contexts in which each may be best suited for a given application. For instance, uncertainty factors may be most useful in cases where a probabilistic assessment is desired but with additional consideration to the strength of knowledge on which the assessment is based, whereas RDM may be more appropriate in contexts where assigning probabilities to uncertain quantities is contentious. The methodologies also demonstrate the ways that risk assessment can inform decision making in conditions where uncertainty and ambiguity make prescriptive approaches inappropriate. In particular, the identification of epistemic uncertainties that most contribute to uncertainty in the resulting risk description or choice of alternatives can provide useful insights into places where additional research or more sophisticated representation could most benefit the assessment. This can ultimately inform more effective responses to deeply uncertain risks such as climate change and support adaptive, deliberative and precautionary approaches to risk management and governance.

\section{ACKNOWLEDGEMENTS}

This research was supported by a National Defense Science and Engineering Graduate Fellowship, by National Science Foundation Grant 1069213 (IGERT), and by the Research Council of Norway through the PETROMAKS2 programme (grant number 228335/E30). This research was conducted while Dr. Shortridge and Dr. Guikema were affiliated with the Department of Geography and Environmental Engineering at Johns Hopkins University. This support is gratefully acknowledged. Any opinions, findings, and conclusions or recommendations expressed in this material are those of the authors and do not necessarily reflect the views of the funding sources.

\section{REFERENCES}

1. Mosleh A, Bier VM. Uncertainty about probability: a reconciliation with the subjectivist viewpoint. IEEE Transactions on Systems, Man and Cybernetics, Part A: Systems and Humans. 1996 May;26(3):303-10.

2. Paté-Cornell ME. Uncertainties in risk analysis: Six levels of treatment. Reliability Engineering \& System Safety. 1996;54(2):95-111.

3. Dubois D. Representation, propagation, and decision issues in risk analysis under incomplete probabilistic information. Risk analysis. 2010;30(3):361-8. 
4. Aven T. A semi-quantitative approach to risk analysis, as an alternative to QRAs. Reliability Engineering \& System Safety. 2008;93(6):790-7.

5. Cox LAT. Confronting Deep Uncertainties in Risk Analysis. Risk Analysis. 2012;32(10):1607-29.

6. Aven T, Zio E. Some considerations on the treatment of uncertainties in risk assessment for practical decision making. Reliability Engineering \& System Safety. 2011;96(1):64-74.

7. Chao PT, Hobbs BF, Venkatesh BN. How climate uncertainty should be included in Great Lakes management: modeling workshop results. Journal of the American Water Resources Association. 1999 Dec;35(6):1485-97.

8. Clark MP, Pulwarty RS. Devising resilient responses to potential climate change impacts. Ogmius: Newsletter of the Center for Science and Technology Policy Research. 2003;5:2-3.

9. Reid SG. Acceptable risk. Engineering safety. 1992;138-66.

10. Mastrandrea MD, Field CB, Stocker TF, Edenhofer O, Ebi KL, Frame DJ, et al. Guidance note for lead authors of the IPCC fifth assessment report on consistent treatment of uncertainties. Intergovernmental Panel on Climate Change (IPCC). 2010;

11. Kaplan S, Garrick BJ. On the quantitative definition of risk. Risk analysis. 1981;1(1):11-27.

12. Walley P. Statistical reasoning with imprecise probabilities. Chapman and Hall London; 1991.

13. Ferson S, Ginzburg LR. Different methods are needed to propagate ignorance and variability. Reliability Engineering \& System Safety. 1996;54(2):133-44.

14. Dubois D, Prade HM, Farreny H, Martin-Clouaire R, Testemale C. Possibility theory: an approach to computerized processing of uncertainty. Vol. 2. Plenum press New York; 1988.

15. Lempert RJ, Groves DG, Popper SW, Bankes SC. A general, analytic method for generating robust strategies and narrative scenarios. Management science. 2006;52(4):514-28.

16. Ben-Haim Y. Robust rationality and decisions under severe uncertainty. Journal of the Franklin Institute. 2000;337(2):171-99.

17. Karvetski CW, Lambert JH. Evaluating deep uncertainties in strategic priority-setting with an application to facility energy investments. Systems Engineering. 2012 Dec;15(4):483-93.

18. Hamilton MC, Lambert JH, Connelly EB, Barker K. Resilience analytics with disruption of preferences and lifecycle cost analysis for energy microgrids. Reliability Engineering \& System Safety. 2016 Jun;150:11-21.

19. Dubois D, Prade H. On the relevance of non-standard theories of uncertainty in modeling and pooling expert opinions. Reliability Engineering \& System Safety. 1992;36(2):95-107.

20. Soundappan P, Nikolaidis E, Haftka RT, Grandhi R, Canfield R. Comparison of evidence theory and Bayesian theory for uncertainty modeling. Reliability engineering \& System safety. 2004;85(1):295-311.

21. Hall JW, Lempert RJ, Keller K, Hackbarth A, Mijere C, McInerney DJ. Robust Climate Policies Under Uncertainty: A Comparison of Robust Decision Making and Info-Gap Methods. Risk Analysis: An International Journal. 2012;32(10):1657-72.

22. Lempert RJ, Collins MT. Managing the Risk of Uncertain Threshold Responses: Comparison of Robust, Optimum, and Precautionary Approaches. Risk Analysis. 2007;27(4):1009-26. 
23. Flage R, Baraldi P, Zio E, Aven T. Probability and Possibility-Based Representations of Uncertainty in Fault Tree Analysis: Comparison of Probabilistic/Possibilistic Computational Approaches. Risk Analysis. 2013 Jan;33(1):121-33.

24. Aven T, Zio E, Baraldi P, Flage R. Uncertainty in Risk Assessment: The Representation and Treatment of Uncertainties by Probabilistic and Non-probabilistic Methods. John Wiley \& Sons; 2013.

25. Bedford T, Cooke R. Probabilistic risk analysis: foundations and methods. Cambridge University Press; 2001.

26. Aven T. Foundations of risk analysis. 2nd ed. Hoboken, N.J: Wiley; 2012.

27. Flage R, Aven T. Expressing and communicating uncertainty in relation to quantitative risk analysis. Reliability \& Risk Analysis: Theory \& Application. 2009;2(13):9-18.

28. Williamson RC, Downs T. Probabilistic arithmetic. I. Numerical methods for calculating convolutions and dependency bounds. International Journal of Approximate Reasoning. 1990;4(2):89-158.

29. Tucker WT, Ferson S. Probability bounds analysis in environmental risk assessment. Setauket, New York: Applied Biomathematics; 2003.

30. Lempert R. Scenarios that illuminate vulnerabilities and robust responses. Climatic Change. 2013;1-20.

31. Friedman JH, Fisher NI. Bump hunting in high-dimensional data. Statistics and Computing. 1999;9(2):123-43.

32. Bryant BP, Lempert RJ. Thinking inside the box: A participatory, computer-assisted approach to scenario discovery. Technological Forecasting and Social Change. 2010;77(1):34-49.

33. Berner C, Flage R. Strengthening quantitative risk assessments by systematic treatment of uncertain assumptions. Reliability Engineering \& System Safety. 2015 Oct;

34. Keynes JM. Chapter IV: The Principle of Indifference. In: A Treatise on Probability. Macmillan and Company, limited; 1921. p. 41-64.

35. Jaynes ET. Information Theory and Statistical Mechanics. Phys Rev. 1957 May 15;106(4):620-30.

36. Ferson S, Tucker WT. Sensitivity in risk analyses with uncertain numbers [Internet]. Sandia National Laboratories Albuquerque, NM; 2006 [cited 2016 Jan 15]. Available from: http://prod.sandia.gov/techlib/access-control.cgi/2006/062801.pdf

37. Linkov I, Satterstrom FK, Kiker G, Batchelor C, Bridges T, Ferguson E. From comparative risk assessment to multi-criteria decision analysis and adaptive management: Recent developments and applications. Environment International. 2006 Dec;32(8):1072-93.

38. Karvetski CW, Lambert JH, Keisler JM, Sexauer B, Linkov I. Climate change scenarios: risk and impact analysis for Alaska coastal infrastructure. International Journal of Risk Assessment and Management. 2011 Jan $1 ; 15(2-3): 258-74$.

39. Aven T. On how to deal with deep uncertainties in a risk assessment and management context. Risk Analysis. 2013;33(12):2082-91.

40. Apostolakis GE. How useful is quantitative risk assessment? Risk analysis. 2004;24(3):515-20.

41. Klinke A, Renn O. A New Approach to Risk Evaluation and Management: Risk-Based, Precaution-Based, and Discourse-Based Strategies1. Risk Analysis. 2002;22(6):1071-94. 
42. Amundrud $\varnothing$, Aven T. On how to understand and acknowledge risk. Reliability Engineering \& System Safety. 2015 Oct;142:42-7.

43. Bjerga T, Aven T. Adaptive risk management using new risk perspectives - an example from the oil and gas industry. Reliability Engineering \& System Safety. 2015 Feb;134:75-82.

44. Lin L, Nilsson A, Sjölin J, Abrahamsson M, Tehler H. On the perceived usefulness of risk descriptions for decision-making in disaster risk management. Reliability Engineering \& System Safety. 2015 Oct;142:48-55.

45. Aven T. On the need for restricting the probabilistic analysis in risk assessments to variability. Risk analysis. 2010;30(3):354-60.

46. Kasprzyk JR, Nataraj S, Reed PM, Lempert RJ. Many objective robust decision making for complex environmental systems undergoing change. Environmental Modelling \& Software. 2013;42:55-71. 


\begin{tabular}{|l|l|l|}
\hline Uncertain Input Quantity & Probabilistic Representation & Underlying Assumption \\
\hline Flood return period & 50 years & Rainfall increases by $15 \%$ \\
\hline $\begin{array}{l}\text { Floodwall failure } \\
\text { probability }\end{array}$ & 0.15 & $\begin{array}{l}\text { Actual probability is close to average } \\
\text { value from city engineers }\end{array}$ \\
\hline Assets at risk & Uniform $\sim(\$ 500 \mathrm{M}, \$ 600 \mathrm{M})$ & $\begin{array}{l}\text { Asset growth rates remain within } \\
\text { historical range }\end{array}$ \\
\hline Construction Costs & Lognormal $\sim(\$ 45 \mathrm{M}, \$ 7 \mathrm{M})$ & $\begin{array}{l}\text { Prices for land and materials remain } \\
\text { consistent with current levels }\end{array}$ \\
\hline
\end{tabular}

\begin{tabular}{|l|l|l|}
\hline & No Action & Upgrades \\
\hline Expected value & $\$ 53.08$ & $\$ 47.99$ \\
\hline 0.025 percentile & $\$ 0$ & $\$ 32.85$ \\
\hline 0.975 percentile & $\$ 576$ & $\$ 61.32$ \\
\hline $\mathrm{P}($ cost $=\$ 0)$ & 0.91 & 0.0 \\
\hline
\end{tabular}

\begin{tabular}{|c|c|c|c|c|}
\hline \multirow[t]{2}{*}{ Assumption } & \multirow{2}{*}{$\begin{array}{l}\text { Quantity } \\
\text { impacted }\end{array}$} & \multirow{2}{*}{$\begin{array}{l}\text { Strength of } \\
\text { Knowledge }\end{array}$} & \multicolumn{2}{|c|}{ Sensitivity } \\
\hline & & & No Action & Upgrades \\
\hline Rainfall increases by $15 \%$ & Flood return period & Weak & Moderate & Low \\
\hline $\begin{array}{l}\text { Failure probability is equal to central } \\
\text { value from engineers }\end{array}$ & $\begin{array}{l}\text { Floodwall failure } \\
\text { probability }\end{array}$ & Moderate & Moderate & None \\
\hline $\begin{array}{l}\text { Asset growth rates remain within } \\
\text { historical range }\end{array}$ & Assets at risk & Strong & Moderate & Low \\
\hline $\begin{array}{l}\text { Land and material costs remain } \\
\text { consistent }\end{array}$ & $\begin{array}{l}\text { Upgrade } \\
\text { construction costs }\end{array}$ & Weak & None & High \\
\hline
\end{tabular}

Table III: Qualitative assessment of uncertainty factors in probabilistic example

\begin{tabular}{|c|c|}
\hline Uncertain Quantity & Representation \\
\hline Flood return period & 20 to 200 years \\
\hline Existing floodwall failure probability & 0.1 to 0.2 \\
\hline Assets at risk & $\$ 500 \mathrm{M}$ to $\$ 600 \mathrm{M}$ \\
\hline Construction costs & 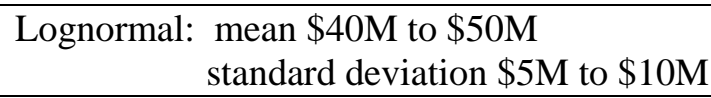 \\
\hline
\end{tabular}

Table IV: Representation of uncertain input quantities in probability bounds assessment 


\begin{tabular}{|l|l|l|}
\hline & No Action & Upgrades \\
\hline Expected value & $\$ 7-\$ 182$ & $\$ 37.12-\$ 63.95$ \\
\hline 0.025 Percentile & $\$ 0-\$ 0$ & $\$ 23.95-\$ 41.05$ \\
\hline 0.975 Percentile & $\$ 0-\$ 1200$ & $\$ 47.18-89.08$ \\
\hline P(total cost $=\$ 0)$ & 0.74 to 0.985 & Negligible \\
\hline
\end{tabular}

Table V: Summary measures for the probability bounds analysis

\begin{tabular}{|l|l|}
\hline Input Quantity & Representation \\
\hline Flood return period & Uniform $(20$ to 200 years $)$ \\
\hline Floodwall failure probability & Existing: Uniform $\sim[0.1$ to 0.2$]$ \\
\hline Assets at risk & Uniform $\sim[\$ 500 \mathrm{M}$ to $\$ 600 \mathrm{M}]$ \\
\hline Upgrade Construction Costs & Lognormal $\sim$ mean: $\$ 45 \mathrm{M}, \mathrm{sd}=\$ 10 \mathrm{M}$ \\
\hline
\end{tabular}

Table VI: Representation of uncertain input quantities in the RDM assessment

\begin{tabular}{|c|c|c|c|}
\hline Method & Uncertainty Factors & Probability Bounds & $\begin{array}{c}\text { Robust Decision } \\
\text { Making }\end{array}$ \\
\hline $\begin{array}{l}\text { Representation } \\
\text { of uncertain } \\
\text { quantities } \\
\end{array}$ & $\begin{array}{l}\text { First order precise } \\
\text { probability distributions }\end{array}$ & $\begin{array}{l}\text { Imprecise probability } \\
\text { distributions }\end{array}$ & $\begin{array}{l}\text { Precise but } \\
\text { uninformative } \\
\text { probability distribution }\end{array}$ \\
\hline $\begin{array}{l}\text { Analytical } \\
\text { output }\end{array}$ & $\begin{array}{l}\text { - Precise distribution of } \\
\text { costs for both alternatives } \\
\text { - Key assumptions } \\
\text { supporting probabilistic } \\
\text { description, their strength } \\
\text { of knowledge and } \\
\text { sensitivity }\end{array}$ & $\begin{array}{l}\text { - Imprecise distribution of } \\
\text { costs for both alternatives }\end{array}$ & $\begin{array}{l}\text { - Scenario describing } \\
\text { conditions where } \\
\text { upgrades result in lower } \\
\text { costs than no-action } \\
\text { - Relative cost of each } \\
\text { alternative across } \\
\text { ensemble of } \\
\text { simulations }\end{array}$ \\
\hline $\begin{array}{l}\text { Risk } \\
\text { management } \\
\text { implications }\end{array}$ & $\begin{array}{l}\text { - Expected costs of } \\
\text { upgrades is slightly lower } \\
\text { than no-action, but with a } \\
\text { lower probability of } \\
\text { incurring very high costs } \\
\text { - Moderate strength of } \\
\text { knowledge } \\
\text { - Key assumptions are } \\
\text { flood return period and } \\
\text { land/material costs }\end{array}$ & $\begin{array}{l}\text { - Expected costs could be } \\
\text { lower for upgrades or no } \\
\text { action } \\
\text { - The probability of very } \\
\text { high costs (above } \$ 500 \mathrm{M}) \\
\text { could be significant for the } \\
\text { no-action alternative } \\
\text { - Epistemic uncertainty has } \\
\text { much larger impact on no- } \\
\text { action than on upgrades }\end{array}$ & $\begin{array}{l}\text { - Key uncertainties } \\
\text { driving decision are } \\
\text { flood return period and } \\
\text { floodwall failure } \\
\text { probability } \\
\text { - No action results in } \\
\text { lower costs than } \\
\text { upgrades in majority } \\
(95 \%) \text { of simulations }\end{array}$ \\
\hline
\end{tabular}

Table VII: Summary of comparison results 


\section{FIGURES}

\section{Risk Assessment Process}

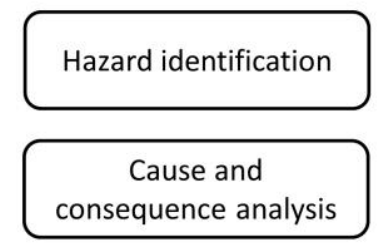

Uncertainty analysis

(generally probabilistic)

\section{Risk Description}

$\left(A^{\prime}, C^{\prime}, Q, K\right)$

$A^{\prime}$ : Specific events

$C^{\prime}$ : Measurement of consequences of $A^{\prime}$

Q: Measurement of uncertainty of $A^{\prime}$ and $C^{\prime}$

K: Knowledge on which $A^{\prime}, C^{\prime}$, and $Q$ are based

Figure 1: Conceptualization of risk assessment process 


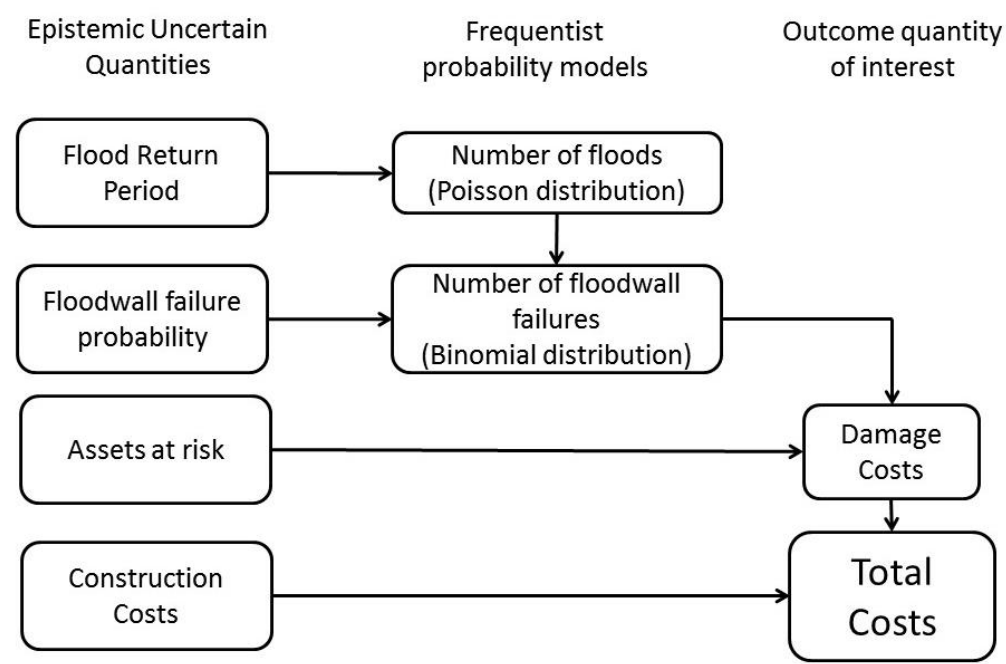

Figure 2: Characterization of uncertain input quantities for flood risk example problem 


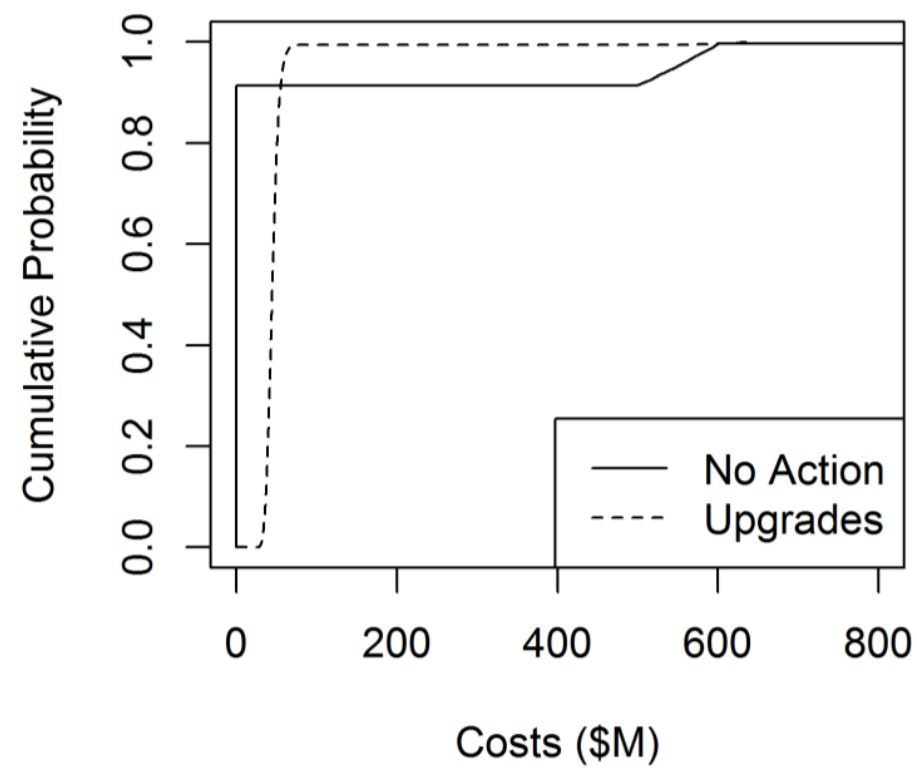

Figure 3: Probability distribution of total costs for no-action and upgrades alternatives 

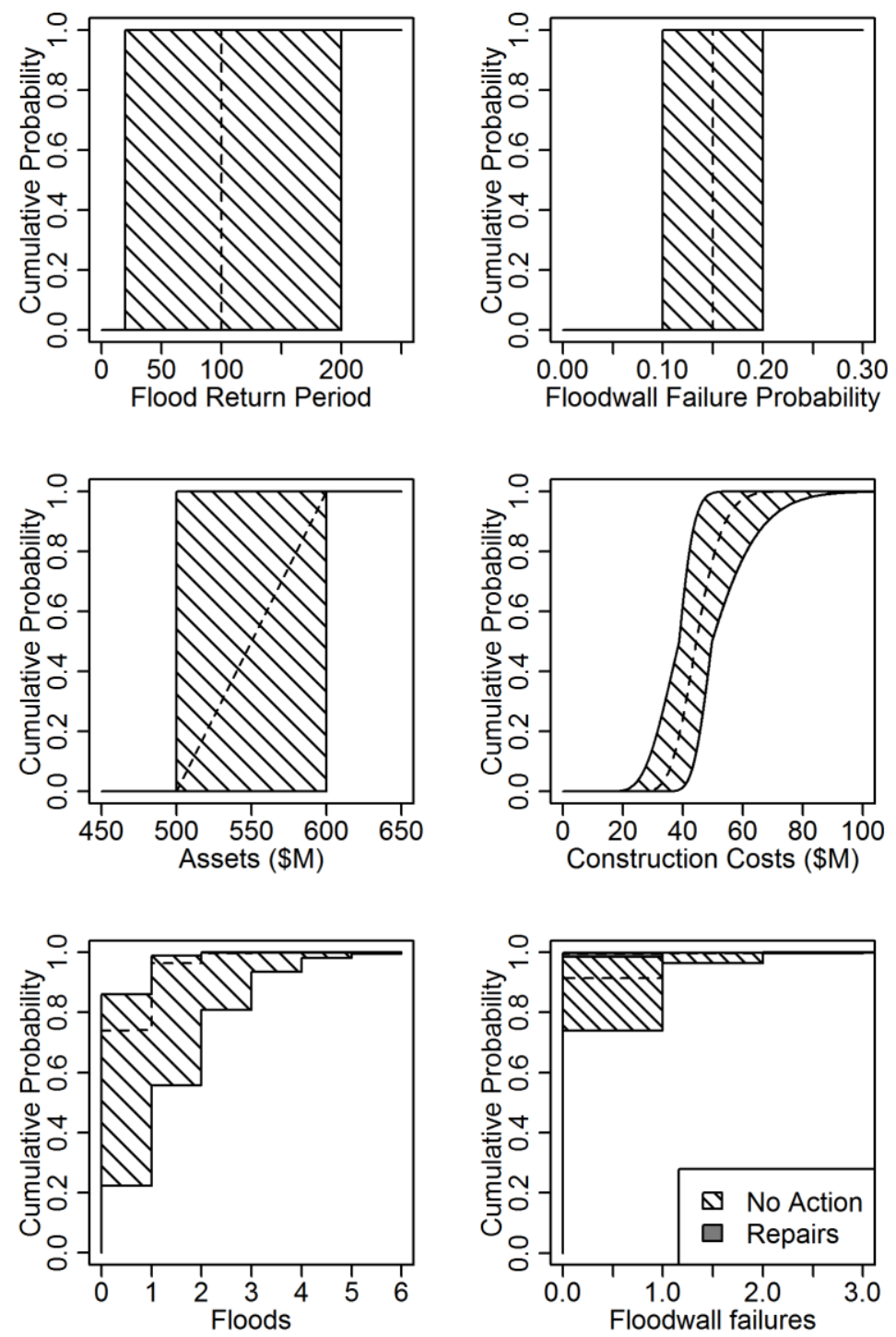

Figure 4: P-boxes for the uncertain input quantities used in the probability bounds assessment. The bottom two figures show p-boxes for the number of floods and the number of floodwall failures for each alternative. Dashed lines show the cumulative distribution function used in the probabilistic assessment. 


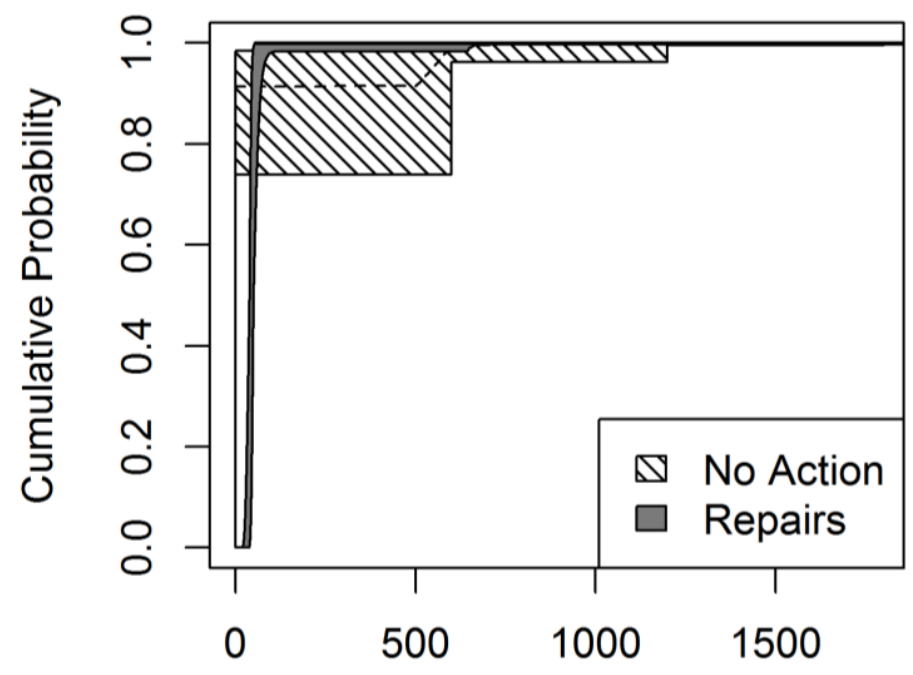

Total Costs (\$M)

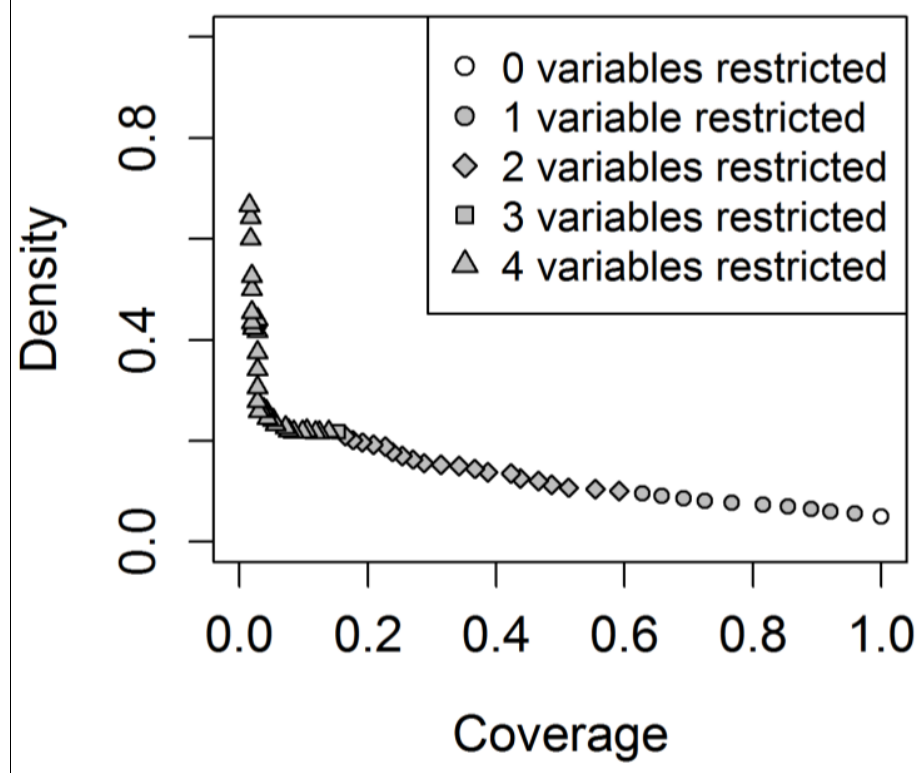

Figure 6: Boxes identified during scenario discovery process for no-action alternative 